\title{
Low dose PTH improves metaphyseal bone healing more when muscles are paralyzed
}

Olof Sandberg, Brandon R Macias and Per Aspenberg

\author{
Linköping University Post Print
}

Tweet

N.B.: When citing this work, cite the original article.

Original Publication:

Olof Sandberg, Brandon R Macias and Per Aspenberg, Low dose PTH improves metaphyseal bone healing more when muscles are paralyzed, 2014, Bone, (63), 15-19.

http://dx.doi.org/10.1016/j.bone.2014.02.008

Copyright: Elsevier

http://www.elsevier.com/

Postprint available at: Linköping University Electronic Press

http://urn.kb.se/resolve?urn=urn:nbn:se:liu:diva-111393 
Low dose PTH improves metaphyseal bone healing more when muscles are paralyzed

Olof Sandberg ${ }^{1 *}$, Brandon R Macias ${ }^{1,2}$, Per Aspenberg ${ }^{1}$

${ }^{1}$ Orthopaedics, Department of Clinical and Experimental Medicine, Linköping University, 58185 Linköping, Sweden

${ }^{2}$ Department of Orthopedic Surgery, University of California, San Diego, USA

*Corresponding author, olof.sandberg@liu.se, +46101034116 


\begin{abstract}
Stimulation of bone formation by PTH is related to mechanosensitivity. The response to PTH treatment in intact bone could therefore be blunted by unloading. We studied the effects of mechanical loading on the response to PTH treatment in bone healing. Most fractures occur in metaphyses, therefor we used a model for metaphyseal bone injury.
\end{abstract}

One hind leg of 20 male SD rats was unloaded via intramuscular botulinum toxin injections. Two weeks later, the proximal unloaded tibia had lost $78 \%$ of its trabecular contents. At this time-point, the rats received bilateral proximal tibiae screw implants. Ten of the 20 rats were given daily injections of $5 \mu \mathrm{g} / \mathrm{kg}$ PTH(1-34). After two weeks of healing, screw fixation was measured by pull-out, and microCT of the distal femur cancellous compartment was performed. Pull-out force provided an estimate for cancellous bone formation after trauma.

PTH more than doubled the pull-out force in the unloaded limbs (from 14 to $30 \mathrm{~N}$ ), but increased it by less than half in the loaded ones (from 30 to $44 \mathrm{~N}$ ). In relative terms, PTH had a stronger effect on pull-out force in unloaded bone than in loaded bone $(\mathrm{p}=0.03)$.

The results suggest that PTH treatment for stimulation of bone healing does not require simultaneous mechanical stimulation.

\title{
Keywords
}

Parathyroid Hormone, fracture healing, metaphyseal fracture, mechanical loading, Botulinum toxin 


\section{Introduction}

Both mechanical loading and parathyroid Hormone (PTH) are important for the maintenance of the structure of uninjured bone. Several studies suggest that the two interact synergistically: with increased levels of PTH, the response to mechanical loading is enhanced and can be induced with less loading [1-4]. In fact, the effects of both PTH and mechanical loading are dependent on L-type voltage-sensitive calcium channels [5].

Also in fracture healing, both mechanical loading and PTH can enhance or accelerate the process, at least in animal models [6]. It has been tempting to suppose that there is a similar synergistic interaction here, as in uninjured bone [7]. This has led to speculations that in human fracture trials, the weight-bearing lower extremity is more likely to respond to PTH treatment, compared to the upper extremity. However, recently Ellegaard et al. found positive effects of both loading and PTH on the healing of tibial shaft fractures in ovariectomized rats, but no synergy between the two. They used intramuscular Botox injections to reduce loading. This data suggests that PTH could be equally useful also under unloaded conditions [8].

Most fractures in humans occur in corticocancellous, metaphyseal bone, and heal to a large extent by new bone formation within the marrow compartment [9]. It can be expected that the biology of shaft fractures and metaphyseal fractures is different, due to the relative abundance of mesenchymal stem cells or progenitors, as well as inflammatory and regulatory cells, in the metaphyseal marrow compartment. In shaft fractures, these cells often have to be recruited from distant sites [10, 11].

We therefore studied the interaction between mechanical loading and PTH treatment in the healing of trabecular bone injury in a rat model. We used male rats, in contrast to Ellegaard et al. who used ovariectomized female rats. Our trauma model has been used in many studies, and comprises insertion of a screw into the metaphysis $[12,13]$. The trabecular fractures caused by the insertion trauma elicit a bone healing response that leads to new bone formation around the screw, gradually increasing fixation strength [14]. The pull-out force at a predetermined time point can therefore serve as a measure of the metaphyseal healing response. 
In contrast to most other studies, we chose a low dose of PTH, estimated to correspond to human clinical use [15].

Our hypothesis was that there would be a significant positive interaction between loading and PTH treatment on the pull-out force in this metaphyseal trauma healing model.

\section{Material and methods}

\subsection{Animals and surgery}

A total of 20 male, 8 weeks old Sprague Dawley rats with a mean weight of $325 \pm 20$ grams were used. All rats were anesthetized with isoflurane gas (Forene, Abbot Scandinavia, Solna, Sweden). Antibiotics (oxytetracycline $25 \mathrm{mg} / \mathrm{kg}$; Engemycin, Intervet, Boxmeer, Holland) were given preoperatively, and analgesics (buprenorphine $0.045 \mathrm{mg} / \mathrm{kg}$; Temgesic, Schering-Plough, Brussels, Belgium) were given both preand postoperatively every 12 hours for 48 hours. The surgery was performed under aseptic conditions.

The rats were given intramuscular Botox injections to induce calf muscle paralysis 2 weeks prior to screw insertion (Botox ${ }^{\circledR}$, Allergan Inc., Irvine, CA). The rats were anesthetized with isoflurane gas and the right hind limb was shaved. Botox was injected into the quadriceps (proximal and distal part), gastrocnemius (lateral and medial) and soleus muscles. Each muscle received $1 \mathrm{U}$, diluted in $0.02 \mathrm{ml}$ saline. The total injected amount per rat was $5 \mathrm{U}$ and $0.1 \mathrm{ml}$.

Screw insertion was conducted 2 weeks after the Botox injections. The rats were again anesthetized with isoflurane. Both legs were shaved, the skin was cleaned, and a sterile draping applied. A hole was drilled in the anterio-medial surface of the proximal tibia of both hind legs, about $3 \mathrm{~mm}$ from the growth plate. A custom made stainless steel screw (thread: M1.7, length of threaded part $2.8 \mathrm{~mm}$ ) was inserted in the metaphyseal bone close to the physis. After surgery, the animals were randomly allocated into two treatment groups $(\mathrm{n}=10)$ and subcutaneously administered either PBS vehicle (control), or Teriparatide (PTH) $5 \mu \mathrm{g} / \mathrm{kg}$ given daily. All injections started the day 
after surgery. Two weeks after surgery the animals were euthanized with carbon dioxide. The tibiae and femora were harvested.

\subsection{Mechanical testing}

The mechanical testing protocol has been described in detail elsewhere [16]. Briefly, steel screws were tested for pull-out strength using a computerized materials testing machine at a cross head speed of $0.1 \mathrm{~mm} / \mathrm{s}$. The bone was fastened in a holder with a 3 $\mathrm{mm}$ diameter hole in which the screw head is placed. The screw is then attached to the material testing machine, and pulled out through the hole. The peak pull-out force was considered the primary variable. During analyses the operator was blinded for treatment.

\section{3 microCT morphometry}

The distal femurs were scanned in a microCT (Skyscan 1174, v. 2, Skyscan, Aarteselar Belgium). An isotropic voxel size of $20 \mu \mathrm{m}$ was used. The scan was performed at $50 \mathrm{kV}$, in 180 degrees, with an aluminum filter of $0.5 \mathrm{~mm}$, and a frame averaging of 3 . The images were reconstructed using NRecon. Corrections for ring artifacts and beam hardening were applied. The volume of interest was defined as the medullary space of the distal femur, starting $1.5 \mathrm{~mm}$ proximal of the growth plate and continuing to $2.5 \mathrm{~mm}$ proximal of the growth plate.

\subsection{Temporal unloading study}

In the main experiment, we delayed implant insertions until 2 weeks after the Botox injection to avoid the phase with drastic changes in bone composition and density that follows the injections. In order to characterize the situation at the time of implant insertion, we performed an additional experiment with Botox injections without surgery or PTH. We unloaded 30 rats unilaterally as described above, and performed bilateral bone morphometry with microCT at baseline and after 2 or 4 weeks. Botox injections, harvest, and microCT measurements were performed as described above, but this time in the proximal tibia.

\subsection{Statistics}

We used the ratio for the pull-out force between the loaded and unloaded sides as predetermined primary effect variable. In order to preclude problems with the 
distribution of these values, due to the use of a ratio, they were compared between PTH and vehicle groups by Mann-Whitney non-parametric test with $95 \%$ CI for differences in group medians calculated according to Hodges-Lehman, using SPSS 20.0. For the microCT analysis, we predetermined the bone volume per total volume (BV/TV) as the main variable, and used Student's t-test after checking for equal variance (Levene's test) and visual inspection of residual distribution.

\section{Results}

\subsection{Exclusions}

One pull-out screw was malpositioned at harvest (Botox PTH), and excluded by the blinded evaluator. The distal femur of another rat (Loaded PTH) was lost at harvest.

\subsection{Mechanical findings}

Comparing the mean value for both legs in non-PTH treated control animals with both legs in PTH treated animals, PTH increased the pull-out force by more than two thirds ( $\mathrm{p}<0.0005$; Table 1). However, the relation between loaded and unloaded legs was different in the two treatment groups. In the control animals, the pull-out force was more than double on the loaded side compared to the unloaded side. In PTH treated animals, the loaded side exhibited a pull-out force less than $50 \%$ higher than the unloaded side (Figures 1 and 2, Table 2). The ratio for the pull-out force between the loaded and unloaded sides was median 2.25 in controls, and 1.41 with PTH (Difference $0.84 ; 95 \%$ CI 0.17 to $1.4 ; \mathrm{p}=0.03$ ). In other words, loading decreased the relative effect of PTH.

\section{3 microCT findings in rats with implants}

In the loaded, uninjured cancellous bone of the distal femur, PTH demonstrated no significant gain of bone total volume (BV/TV). In the unloaded femurs of control rats, the BV/TV had decreased to about a quarter of that in the loaded legs (Table 3, Figure 3). The BV/TV seemed to be slightly increased by PTH, but the difference was not significant ( $\mathrm{p}=0.07$, for the effect of PTH on the Botox legs) and there was no significant effect on the loaded/unloaded ratios. Of the other primary morphometric variables, there was an effect on trabecular number: unloaded legs had fewer 
trabeculae, but their number was increased by PTH ( $p=0.005$, for the effect of PTH on the Botox legs, Table 3).

\subsection{Temporal unloading study}

In the rats without implants, the BV/TV of the cancellous bone of the proximal tibia was reduced by $78 \%$ (95\% CI: 59 to $96 \%$ ) 2 weeks after Botox injection. After 4 weeks the reduction was $85 \%$ (95\% CI 67 to 100\%), (Table 4, Figure 4). On the loaded side, no increase was noted. Instead, the BV/TV was reduced by $38 \%$ (95\% CI 22 to $54 \%$ ) after 2 weeks. There was no change from week 2 to week 4, at which time the reduction was $34 \%$ (95\% CI 16 to $52 \%$ ).

\section{Discussion}

We found that PTH more than doubled the pull-out force in unloaded limbs, but had relatively less effect in the loaded limbs. The pull-out forces in the loaded limbs were not limited by a ceiling effect inherent in the model: much higher forces are possible. With higher doses of PTH we have seen mean pull-out forces that were higher than in any specimen of this study $[17,18]$. We made the present, unexpected findings about a year before by Ellegaard et al. published a study, where they saw no synergistic effects of loading and PTH in shaft fractures in ovariectomized rats [8]. Because our findings deviated from common belief, we temporarily set the project aside, but the article by Ellegaard et al. reinvigorated our efforts to publish these data.

Because sclerostin is thought to be a main player in the response to mechanical load, and sclerostin antibodies appear to have a potent anabolic effect on bone [19], we compared PTH and sclerostin antibodies in the same model as in the current study [20]. That work again suggested that PTH has a specific effect on fracture healing, whereas the effect of the sclerostin antibodies was weak at uninjured sites. These findings, together with those of Ellegaard et al., as well as our current findings, all seem to fit with the possibility that PTH stimulates fracture healing regardless of mechanical loading and by signaling mechanisms independent of Sclerostin.

The dose of PTH (teriparatide) in our study was small $(5 \mu \mathrm{g} / \mathrm{kg})$, and almost all previous rat studies with teriparatide have used several-fold higher doses. However, in 
a dog study of implant integration, small but significant effects were seen with $5 \mu \mathrm{g} / \mathrm{kg}$ [21]. We chose this dose because when we are close to the lowest efficacious dose, the effects of possible contributing factors, such as loading, are more likely to make a difference. Moreover, this dose appears to correspond to the approved human dose, as it has been shown to yield a systemic exposure in rats of about 1.6 to 3 times the clinical dose in humans, as estimated from the area under the curve of serum concentration over time [15].

We chose to give the Botox injections 2 weeks before surgery, to allow screw insertion into bone with reduced loading, rather than a situation with a recently perturbed loading status. The BV/TV measurements from the proximal tibiae in the temporal unloading study confirmed that most of the bone loss due to the Botox injections had already occurred after 2 weeks. We had expected a small increase in bone density on the contralateral side, due to increased, compensatory loading. Instead there was a decrease, probably due to generally decreased physical activity. Furthermore, in the uninjured bone of the femur in operated rats, with continued loading, PTH had no measurable effects. This is to expect, considering the low dose of PTH and the short period of treatment. The effects of unloading were similar to those of the temporal unloading study.

The current results point at the differences between the effects of PTH on background bone remodeling, and on healing after bone trauma. The effects on healing after trauma are much stronger than the effects of on uninjured bone [22]. PTH appears to influence several parts of the fracture healing process, including early cartilage formation [23]. The currently used model, in metaphyseal bone, is dominated by intramembranous bone formation, as cartilage does not appear. Intramembranous bone formation appears to dominate also in human in fractures of cancellous bone [9].

A main limitation of our study, similar to that of Ellegaard et al., was that we studied the effects of reducing loading with Botox, rather than increasing loading by any other means. However, bone loss in response to reductions in mechanical loading is clinically relevant. Because the change in loading status in our model influences the whole proximal tibia and not only the localized injury, it could be argued that the general conditions for bone healing have been compromized by a generally altered mechanical environment in the whole proximal tibia. Another limitation is the use of 
an implant for measuring the response to metaphyseal bone injury. The relevance for screw pull-out fixation as a proxy for cancellous bone healing might be questioned. However, it must be noted that the screw is not osseointegrated, as it is made of steel, not titanium. Therefore, the pull-out force is not dependent on any form of bond between the implant and the bone, but on the quality and quantity of the bone in and around the screw threads. The pull-out force immediately after insertion is low, and then increases gradually, when the healing response to the injury of insertion leads to new bone formation [14]. For the screw to be pulled out, this new bone has to be broken, and the pull-out force therefore provides a measure of its strength. Three-point bending studies or mechanical compression tests of cancellous biopsies in future experiments may provide additional information. However, in our opinion, this is the best currently available model to test cancellous bone trauma healing in an animal model.

Another limitation is that we used rats that were only a little more than 2 months old and still growing rather fast. Tibial growth at this age is about $0.5 \mathrm{~mm}$ per week (own unpublished data). The site of implantation was therefore probably undergoing more intense remodeling than in the adult skeleton, and our results can't be generalized to skeletally mature individuals. However, male rats grow continuously, and this limitation might be relevant for all bone experiments using rats.

The use of botulinum toxin for mechanical unloading has become increasingly popular [24]. Botulinum toxin selectively blocks peripheral cholinergic signaling, i.e. in this context the neuromuscular junctions [25]. It is unlikely that any intraosseous structures would have been directly influence by the injections even if there had been leakage from the injected muscles.

In osteoporosis treatment, PTH appears to have somewhat different effects at different skeletal regions, possibly in part due to different loading status [26]. It has therefore been thought that if studies are designed to show the efficacy of PTH for stimulation of fracture healing, the lower extremities should be preferred. However, the current results suggest that the effects of PTH on healing after metaphyseal bone trauma do not require mechanical loading and can even compensate for lack of mechanical stimulation. 


\section{Acknowledgments}

The study was funded by the Swedish Research Council (VR 02031-47-5), Linköping University, Östergötland County Council, and the King Gustaf V and Queen Victoria Free Mason Foundation. National Science Foundation (NSF) Graduate Research Fellowship Program and NSF/Swedish Research Council — Nordic Research Opportunity (to BRM). 


\begin{tabular}{|l|l|l|}
\hline & & Mean (SD) \\
\hline \multirow{2}{*}{$\begin{array}{l}\text { Vehicle } \\
n=10\end{array}$} & Unload & $13.7(3.4)$ \\
\cline { 2 - 3 } & Load & $29.8(10)$ \\
\hline \multirow{2}{*}{$\begin{array}{l}\text { PTH } \\
n=10\end{array}$} & Unload & $30.3(6.4)$ \\
\cline { 2 - 3 } & Load & $44.3(12)$ \\
\hline
\end{tabular}

Table 1. Pull-out force (N) for metaphyseal stainless steel screws 2 weeks after insertion. Note that unloaded and loaded implants were paired.

\begin{tabular}{|l|l|l|l|l|}
\hline & $\mathrm{n}$ & Median & Min & Max \\
\hline Vehicle & 10 & 2.25 & .92 & 3.58 \\
\hline PTH & 9 & 1.41 & .94 & 2.23 \\
\hline
\end{tabular}

Table 2. Pull-out force: Ratio Loaded/Unloaded. Data from table 1.

\begin{tabular}{|l|l|l|l|l|}
\hline & & $\begin{array}{l}\text { BV/TV } \\
\text { Mean (SD) }\end{array}$ & $\begin{array}{l}\text { TbN } \\
\text { Mean (SD) }\end{array}$ & $\begin{array}{l}\text { MbTh } \\
\text { Mean (SD) }\end{array}$ \\
\hline $\begin{array}{l}\text { Vehicle } \\
\mathrm{n}=10\end{array}$ & Unload & $9.2(4)$ & $0.92(0.4)$ & $0.10(0.005)$ \\
\cline { 2 - 5 } & Load & $35(5)$ & $2.9(0.4)$ & $0.12(0.003)$ \\
\hline $\begin{array}{l}\text { PTH } \\
n=10\end{array}$ & Unload & $13(4)$ & $1.2(0.4)$ & $0.11(0.004)$ \\
\cline { 2 - 5 } & Load & $37(4)$ & $3.0(0.3)$ & $0.12(0.002)$ \\
\hline
\end{tabular}

Table 3. microCT measurement of cancellous bone architecture in the distal femur . Note that unloaded and loaded sides were paired. 


\begin{tabular}{|l|l|l|l|l|l|l|}
\hline & \multicolumn{3}{|c|}{ Unload } & \multicolumn{3}{c|}{ Load } \\
\hline & $\begin{array}{l}\text { BV/TV } \\
\text { Mean (SD) }\end{array}$ & $\begin{array}{l}\text { BMD } \\
\text { Mean (SD) }\end{array}$ & $\begin{array}{l}\text { TbTh } \\
\text { Mean (SD) }\end{array}$ & $\begin{array}{l}\text { BV/TV } \\
\text { Mean (SD) }\end{array}$ & $\begin{array}{l}\text { BMD } \\
\text { Mean (SD) }\end{array}$ & $\begin{array}{l}\text { MbTh } \\
\text { Mean (SD) }\end{array}$ \\
\hline Week 0 & $18(5)$ & $0.24(0.04)$ & $0.046(0.001)$ & $19(4)$ & $0.24(0.03)$ & $0.047(0.001)$ \\
\hline Week 2 & $4.1(2)$ & $0.11(0.02)$ & $0.047(0.002)$ & $12(2)$ & $0.18(0.02)$ & $0.051(0.001)$ \\
\hline Week 4 & $2.7(1)$ & $0.084(0.02)$ & $0.050(0.003)$ & $13(3)$ & $0.18(0.03)$ & $0.052(0.003)$ \\
\hline
\end{tabular}

Table 4. microCT measurements of cancellous bone of the proximal tibia. Unloaded and loaded sides were paired, $\mathrm{n}=10$ for each group.

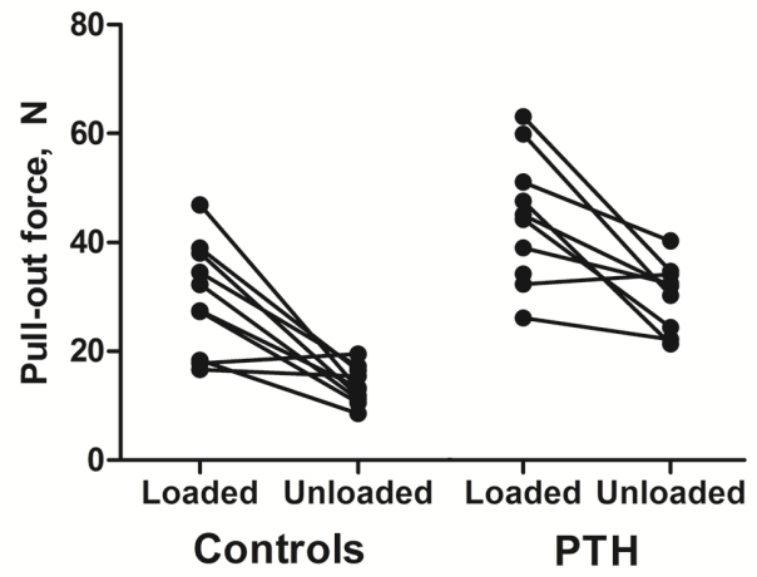

Fig 1. Pull-out force $(\mathrm{N})$ for metaphyseal stainless steel screws 2 weeks after insertion. Loaded and unloaded implants in the same animal connected with lines. 


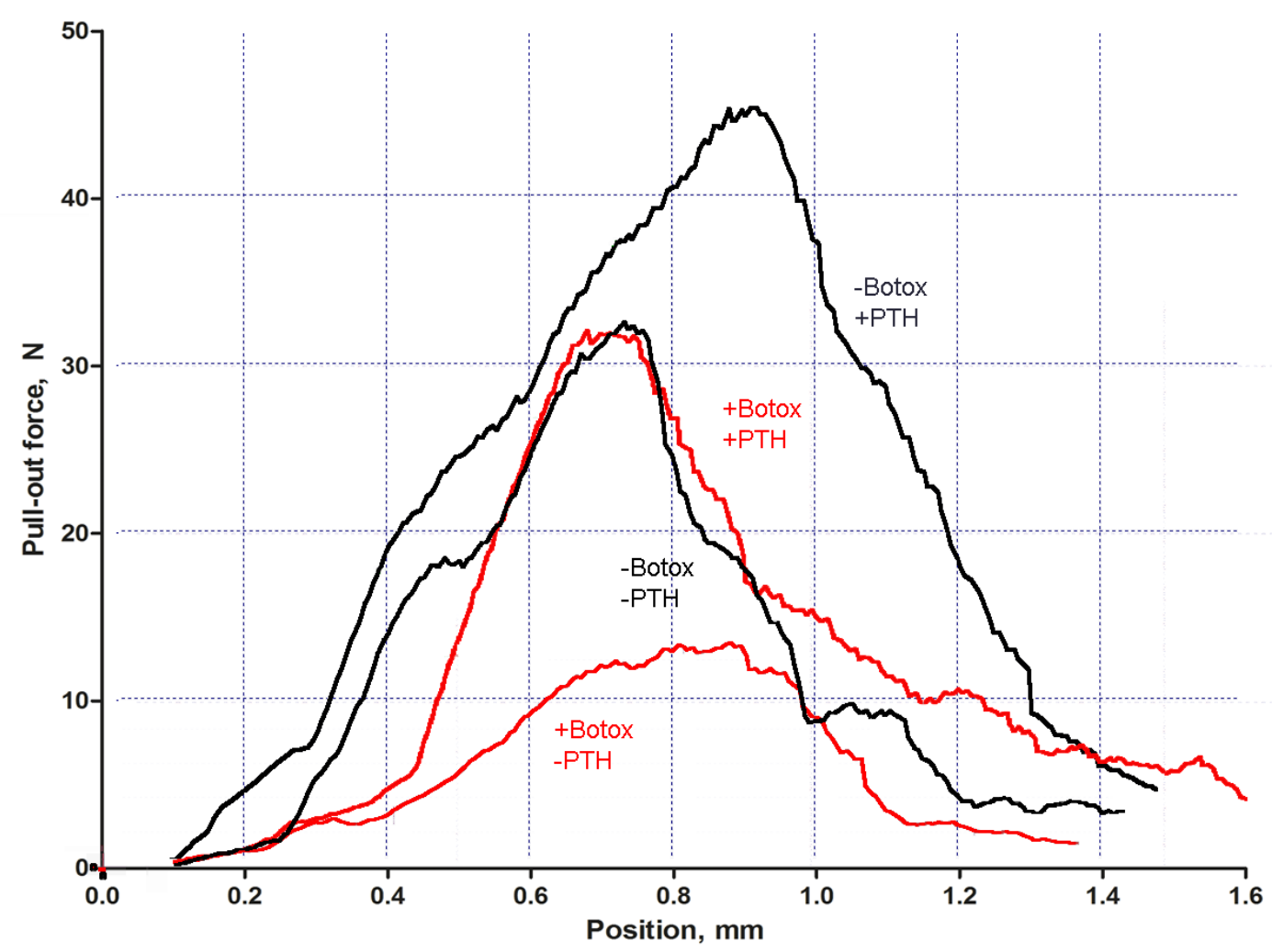

Fig 2. Pull-out force diagrams. Each curve represents the specimen with the median value for peak force in the 4 groups. The curves do not show the characteristic shape of most tension tests, partly because of some motion in the fixation device, partly because of small fractures occurring before the final failure. Therefore, peak force is used as the main variable.

\section{No Botox}

No PTH

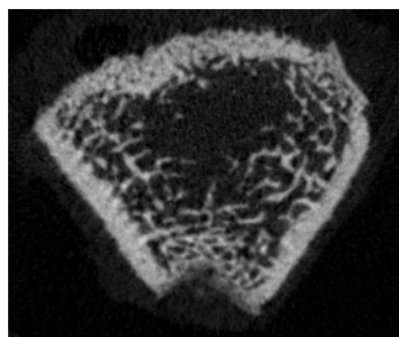

Botox
PTH
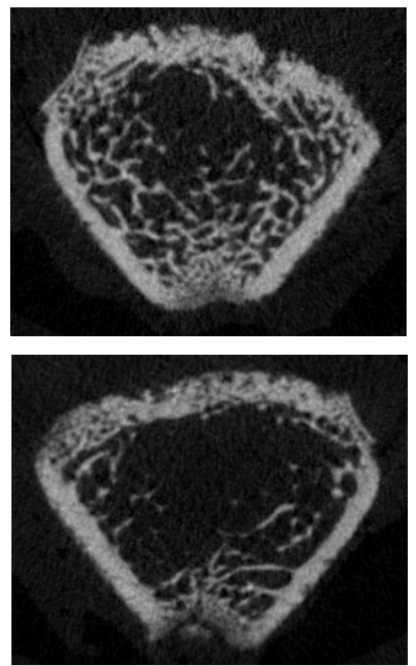
Fig 3. microCT pictures showing a distal femur from each treatment group. The specimens are the ones with the median BV/TV in each group.

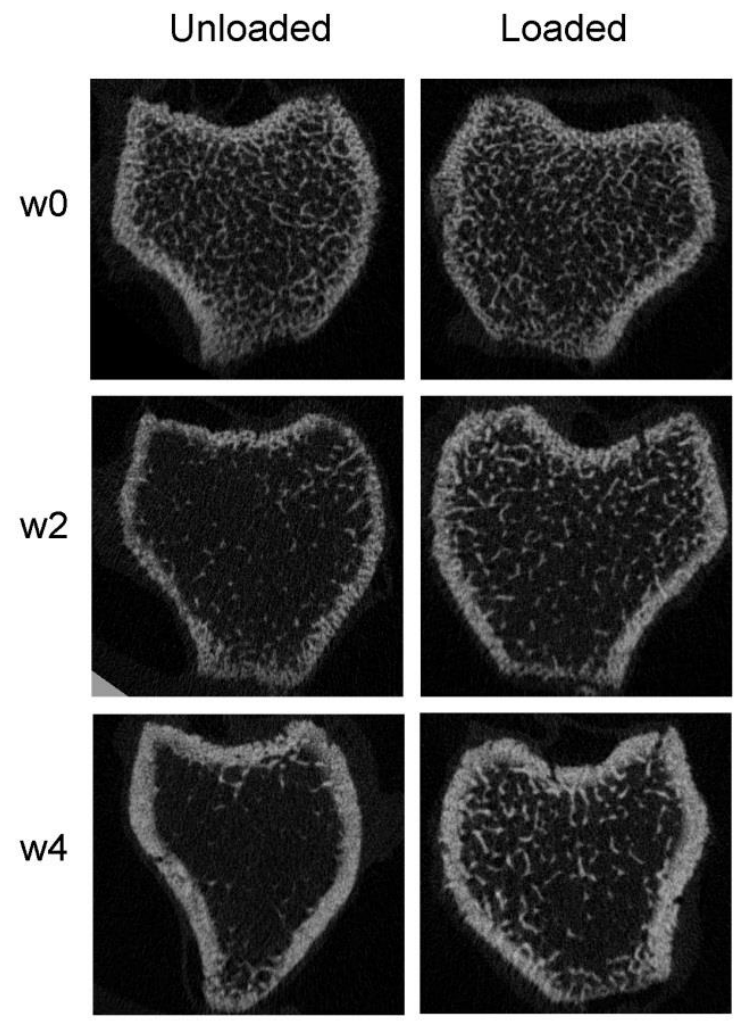

Fig 4. microCT of untraumatized proximal tibia with or without Botox and harvested at 0,2 , and 4 weeks. The specimens are the ones with the median BV/TV in each group, sectioned at the same distance from epiphysis 


\section{References}

[1] Chow JW, Fox S, Jagger CJ, Chambers TJ. Role for parathyroid hormone in mechanical responsiveness of rat bone. Am J Physiol 1998;274: E146-54.

[2] Turner RT, Lotinun S, Hefferan TE, Morey-Holton E. Disuse in adult male rats attenuates the bone anabolic response to a therapeutic dose of parathyroid hormone. J Appl Physiol (1985) 2006;101: 881-6.

[3] Sugiyama T, Saxon LK, Zaman G, Moustafa A, Sunters A, Price JS, Lanyon LE. Mechanical loading enhances the anabolic effects of intermittent parathyroid hormone (1-34) on trabecular and cortical bone in mice. Bone 2008;43: 238-48.

[4] Roberts MD, Santner TJ, Hart RT. Local bone formation due to combined mechanical loading and intermittent hPTH-(1-34) treatment and its correlation to mechanical signal distributions. J Biomech 2009;42: 2431-8.

[5] Li J, Duncan RL, Burr DB, Gattone VH, Turner CH. Parathyroid hormone enhances mechanically induced bone formation, possibly involving L-type voltage-sensitive calcium channels. Endocrinology 2003;144: 1226-33.

[6] Aspenberg P. Annotation: parathyroid hormone and fracture healing. Acta Orthop 2013;84: 4-6.

[7] Gardner MJ, van der Meulen MC, Carson J, Zelken J, Ricciardi BF, Wright TM, Lane JM, Bostrom MP. Role of parathyroid hormone in the mechanosensitivity of fracture healing. J Orthop Res 2007;25: 1474-80.

[8] Ellegaard M, Kringelbach T, Syberg S, Petersen S, Beck Jensen JE, Bruel A, Jorgensen NR, Schwarz P. The effect of PTH(1-34) on fracture healing during different loading conditions. J Bone Miner Res 2013;28: 2145-55.

[9] Aspenberg P, Sandberg O. Distal radial fractures heal by direct woven bone formation. Acta Orthop 2013;84: 297-300.

[10] Kumagai K, Vasanji A, Drazba JA, Butler RS, Muschler GF. Circulating cells with osteogenic potential are physiologically mobilized into the fracture healing site in the parabiotic mice model. J Orthop Res 2008;26: 165-75.

[11] Liu R, Birke O, Morse A, Peacock L, Mikulec K, Little DG, Schindeler A. Myogenic progenitors contribute to open but not closed fracture repair. BMC Musculoskelet Disord 2011;12: 288.

[12] Skripitz R, Aspenberg P. Parathyroid hormone--a drug for orthopedic surgery? Acta Orthop Scand 2004;75: 654-62.

[13] Aspenberg P. Bisphosphonates and implants: an overview. Acta Orthop 2009;80: 119-23.

[14] Wermelin K, Tengvall P, Aspenberg P. Surface-bound bisphosphonates enhance screw fixation in rats--increasing effect up to 8 weeks after insertion. Acta Orthop 2007;78: 385-92.

[15] Tashjian AH, Jr., Chabner BA. Commentary on clinical safety of recombinant human parathyroid hormone 1-34 in the treatment of osteoporosis in men and postmenopausal women. J Bone Miner Res 2002;17: 1151-61.

[16] Sandberg O, Eliasson P, Andersson T, Agholme F, Aspenberg P. Etanercept does not impair healing in rat models of tendon or metaphyseal bone injury. Acta orthopaedica 2012;83: 305-10.

[17] Aspenberg P, Wermelin K, Tengwall P, Fahlgren A. Additive effects of PTH and bisphosphonates on the bone healing response to metaphyseal implants in rats. Acta orthopaedica 2008;79: 111-5.

[18] Agholme F, Macias B, Hamang M, Lucchesi J, Adrian MD, Kuhstoss S, Harvey A, Sato M, Aspenberg P. Efficacy of a sclerostin antibody compared to a low 
dose of PTH on metaphyseal bone healing. Journal of orthopaedic research : official publication of the Orthopaedic Research Society 2014;32: 471-6.

[19] Agholme F, Aspenberg P. Wnt signaling and orthopedics, an overview. Acta Orthop 2011;82: 125-30.

[20] Agholme F, Macias B, Hamang M, Lucchesi J, Adrian MD, Kuhstoss S, Harvey A, Sato M, Aspenberg P. Efficacy of a sclerostin antibody compared to a low dose of PTH on metaphyseal bone healing. J Orthop Res 2013.

[21] Daugaard H, Elmengaard B, Andreassen TT, Lamberg A, Bechtold JE, Soballe K. Systemic intermittent parathyroid hormone treatment improves osseointegration of press-fit inserted implants in cancellous bone. Acta Orthop 2012;83: 411-9.

[22] Skripitz R, Andreassen TT, Aspenberg P. Strong effect of PTH (1-34) on regenerating bone: a time sequence study in rats. Acta Orthop Scand 2000;71: 619-24.

[23] Alkhiary YM, Gerstenfeld LC, Krall E, Westmore M, Sato M, Mitlak BH, Einhorn TA. Enhancement of experimental fracture-healing by systemic administration of recombinant human parathyroid hormone (PTH 1-34). J Bone Joint Surg Am 2005;87: 731-41.

[24] Virchenko O, Aspenberg P. How can one platelet injection after tendon injury lead to a stronger tendon after 4 weeks? Interplay between early regeneration and mechanical stimulation. Acta orthopaedica 2006;77: 806-12.

[25] Dolly JO, Aoki KR. The structure and mode of action of different botulinum toxins. European journal of neurology : the official journal of the European Federation of Neurological Societies 2006;13 Suppl 4: 1-9.

[26] Yu EW, Neer RM, Lee H, Wyland JJ, de la Paz AV, Davis MC, Okazaki M, Finkelstein JS. Time-dependent changes in skeletal response to teriparatide: escalating vs. constant dose teriparatide (PTH 1-34) in osteoporotic women. Bone 2011;48: 713-9. 